\title{
Cost-Effectiveness Analysis of Cervical Anterior Fusion and Cervical Artificial Disc Replacement in the Korean Medical System
}

\author{
Hyosang Lee, M.D., ${ }^{1}$ Ui Chul Kim, M.P.H., Jae Keun Oh, M.D., Ph.D., ${ }^{3}$ Taehyun Kim, Ph.D., ${ }^{4}$ Sohee Park, Ph.D., ${ }^{4}$ \\ Yoon Ha, M.D., Ph.D. ${ }^{2}$ \\ Serim Hospital, Incheon, Korea \\ Department of Neurosurgery, ${ }^{2}$ Severance Hospital, Yonsei University College of Medicine, Seoul, Korea \\ Spine Center, ${ }^{3}$ Department of Neurosurgery, Hallym University Sacred Heart Hospital, Anyang, Korea \\ Graduate School of Public Medicine, ${ }^{4}$ Yonsei University, Seoul, Korea
}

Objective : This study is a retrospective cost-benefit analysis of cervical anterior interbody fusion and cervical artificial disc replacement, which are the main surgical methods to treat degenerative cervical disc disease.

Methods : We analyzed 156 patients who underwent anterior cervical disc fusion and cervical artificial disc replacement from January 1, 2008 to December 31, 2009, diagnosed with degenerative cervical disc disorder. In this study, the costs and benefits were analyzed by using quality adjusted life year (QALY) as the outcome index for patients undergoing surgery, and a Markov model was used for the analysis. Only direct medical costs were included in the analysis; indirect medical costs were excluded. Data were analyzed with TreeAge Pro $2015^{\mathrm{TM}}$ (TreeAge Software, Inc, Williamstown, MA, USA).

Results : Patients who underwent cervical anterior fusion had a total cost of KRW 2501807/USD 2357 over 5 years and obtained a utility of 3.72 QALY. Patients who underwent cervical artificial disc replacement received 4.18 QALY for a total of KRW 3685949/USD 3473 over 5 years. The cumulative cost-effectiveness ratio of cervical spine replacement surgery was KRW 2549511/QALY (USD 2402/QALY), which was lower than the general Korean payment standard.

Conclusion : Both cervical anterior fusion and cervical artificial disc replacement are cost-effective treatments for patients with degenerative cervical disc disease. Cervical artificial disc replacement may be an effective alternative to obtain more benefits.

Key Words : Cost-effectiveness analysis · Quality-adjusted life years · Cervical vertebrae · Total disc replacement · Spinal fusion.

\section{INTRODUCTION}

The number of patients receiving treatment for degenerative cervical disease is increasing year by year, and the annual growth rate is also increasing (National Health Insurance Corporation, 2013). The pain and dysfunction that may be

-Received : April 3, 2018 •Revised : April 18, 2018 •Accepted : May 23, 2018

- Address for reprints : Yoon Ha, M.D., Ph.D.

Department of Neurosurgery, Severance Hospital, Yonsei University College of Medicine, 50-1 Yonsei-ro, Seodaemun-gu, Seoul 03722, Korea Tel : +82-2-2228-2150, Fax : +82-2-393-9979, E-mail : hayoon@yuhs.ac

This is an Open Access article distributed under the terms of the Creative Commons Attribution Non-Commercial License (http://creativecommons.org/licenses/by-nc/4.0) which permits unrestricted non-commercial use, distribution, and reproduction in any medium, provided the original work is properly cited. 
caused by cervical nerve compression can be conservatively treated with anti-inflammatory drugs and physical therapy or injection. If pain or dysfunction continues despite these conservative treatments, anterior cervical discectomy and fusion (ACDF) may be performed ${ }^{4,8)}$. ACDF shows a high recovery rate of over $90 \%$ for patients with radiculopathy and myelopathy $^{2)}$. However, because ACDF completely fuses the cervical vertebrae, exercise function is lost. This loss increases the load on adjacent abutments, thereby exacerbating the degenerative changes and resulting in adjacent segment disease ${ }^{7)}$.

An alternative to ACDF is cervical total disc replacement (cTDR). This surgical method can preserve motion by inserting an artificial disc. Consequently, it can reduce the load on adjacent segments and reduce the incidence of adjacent segment disease. In addition, the cervical disc height and alignment can be adjusted ${ }^{1,3)}$.

In the United States, the cost and benefits of both surgeries have been studied. Because of its inclusion in the diagnosis related group (DRG), the cost of medical care for cTDR is lower than that for ACDF. cTDRs have been reported to be more cost effective than ACDFs ${ }^{10)}$. However, in Korea, the cost of surgery and materials used for cTDR are higher than those of ACDF (Health Insurance Review and Evaluation Center, 2016). Therefore, we cannot conclude that cTDR is more cost effective than ACDF in Korea by applying the US research results. Therefore, the costs and benefits of cTDR and ACDF should be studied in the Korean medical system.

The purpose of this study was to conduct a cost-effectiveness analysis of ACDF and cTDR.

\section{MATERIALS AND METHODS}

This study is a retrospective cost-effectiveness analysis of ACDF and cTDR, which are two alternative surgical methods to treat degenerative cervical disc disease. A virtual cohort was set up to perform a comparison-utility analysis for each method. The virtual cohort was a 40 -year-old patient with degenerative cervical disc disease who received surgical treatment.

We selected 156 patients who underwent ACDF and cTDR surgery at Severance Hospital in Seoul from January 1, 2008 to December 31, 2009 for the probability of metastasis and cost estimation in each Markov model. Five years of medical records were reviewed. The selection and exclusion criteria were as follows. Selection criteria : 1) men and women aged between 30 and 50 years and 2) patients who received singlesegment ACDF or single-segment cTDR due to degenerative cervical disc disease; exclusion criteria : 1) patients with a history of cervical surgery, 2) patients who received ACDF or cTDR through hospitalization due to other diseases, 3) patients with cervical disease due to trauma (within 1 year before surgery, including traffic accidents), 4) patients with tuberculosis-induced cervical disease, 5) ossification of the posterior longitudinal ligament, and 6) cerebral palsy.

The general characteristics of the study group are shown in Table 1. In addition to ACDF and CTDR, there are various other surgical procedures for patients with degenerative cervical disc disease. However, the subjects of this study had singlesegment degenerative cervical disc disease, and patients with segmental degenerative cervical disc disease are usually treated with ACDF and CDR. Other procedures were not considered. The study was reviewed and approved by the Institutional Review Board of Yonsei University Severance Hospital (IRB No. 4-2016-0061).

In this study, the analysis cycle was 1 year, and the Markov model was set to repeat every year. During the analysis period, a model was constructed to perform a cost-benefit analysis based on the number of people in each health state, quality adjusted life year (QALY), and cost for each cycle.

To calculate the transition probability and cost to each health state from the Markov model, subjects' medical records for 5 years after surgery were tracked. The analysis period of this study was 5 years, so the cohort of 40 years old was analyzed until 45 years old. The model composition and analysis were performed with TreeAge Pro $2015^{\mathrm{TM}}$ (TreeAge Software, Inc, Williamstown, MA, USA).

Health status was classified in the Markov model according

Table 1. General subject characteristics

\begin{tabular}{lcc}
\hline & ACDF & cTDR \\
\hline Number of subjects (male/female) & $93(53 / 40)$ & $63(36 / 27)$ \\
Age 30 s & 18 & 24 \\
Age 40 s & 29 & 20 \\
Age 50 s & 46 & 19 \\
Average age & 48 & 44 \\
\hline
\end{tabular}

ACDF : anterior cervical discectomy and fusion, CTDR : cervical total disc replacement 
to the occurrence of complications after ACDF and cTDR surgery. Complications after ACDF and cTDR surgery were classified into reoperations and pain control procedures. Treatment for pain control refers to an injection method rather than surgery. After the surgery, patients were classified as not having complications if their symptoms decreased or disappeared and no further treatment was necessary or if pain or other symptoms remained but were tolerable and did not disrupt daily living. The patients were classified as having complications if they underwent procedures or surgery due to unacceptable pain or other symptoms. We assumed that all reoperations were ACDF procedures because only ACDF was received. The definition of each condition is shown in Table 2.

The transition process of ACDF and cTDR in each Markov model was as follows. Patients with degenerative cervical disc disease who underwent single segment ACDF surgery were divided into two groups : patients who did not require treatment, such as surgery or pain procedure, because they could tolerate symptoms or symptoms disappeared after surgery and patients who had symptoms that could not be tolerated after surgery and needed pain procedures.

The transition process of the cTDR group was almost the same as that of the ACDF group. The second operation was assumed to be ACDF, not cTDR.

The Markov model that reflects the two processes is shown in Fig. 1. The Markov model was used to estimate the transition probability of each health state based on data obtained from medical records. Because each health state was classified according to complications after ACDF and cTDR, the probability of each type of complication and the transition probability to each health condition are the same.

We examined records of reoperations or pain procedures in the 156 enrolled patients. The probability of transition to each health condition is shown in Table 3.

In the medical field, cost-effectiveness analysis experts have used a discount rate of 3\% in the United States, and many studies have been conducted with discount rate of $3 \%{ }^{6}$. Qureshi et al. ${ }^{10)}$ and McAnany et al. ${ }^{9)}$ also applied a discount rate of 3\% for cost-utility analyses with the same research objectives as in this study. Therefore, we used a discount rate of $3 \%$.

Medical costs were divided into the cost of surgery and the cost of complications. Costs incurred due to complications were divided into procedure (injection) costs and reoperation cost. Surgery and reoperation costs included all medical costs incurred during the hospitalization for surgery and reoperation. We did not consider any non-medical costs. Outpatient costs incurred while visiting the hospital for periodic medical care after surgery were excluded. The average cost was used because the costs incurred varied according to the type of procedure. The operation cost and reoperation cost were calculated as an average for all patients. The medical costs are shown in Table 4.

In this study, QALY was used as the variable for utility analysis. The factors needed to estimate QALY are survival and utility weights. ACDF and cTDR were assumed to have no effect on survival during the analysis period of 5 years.

To estimate utility weights, we reviewed questionnaires, but the data were not available. No studies have reported utility weights of patients with degenerative cervical disc disease in foreign literature. However, according to Fryback et al. ${ }^{5)}$ and Tengs and Wallace ${ }^{11)}$, the utility weight for 'condition with arthritis in any joint' was defined as 0.7. In this study, utility weight for degenerative disc disease, which is the starting con-

Table 2. Definition of health status

\begin{tabular}{ll}
\hline Health status & DDD requiring surgical treatment \\
\hline DDD & Patients who underwent ACDF \\
CDF & Patients who underwent cTDR \\
CTDR & Patients whose symptoms disappeared or were tolerable and needed no further treatment \\
Disappear or tolerable $S x$ & Patient whose symptoms were still present or were not tolerable and needed a pain procedure \\
Intolerable Sx and pain procedure & Patients whose symptoms disappeared after a pain procedure and needed no further treatment \\
Disappear or tolerable Sx 2 & Patients who received ACDF again \\
\hline People who had a revision ACDF & \\
\hline DDD : degenerative disc disease, CDF : cervical disectomy and fusion, ACDF : anterior cervical discectomy and fusion, cTDR : cervical total disC \\
replacement, Sx: symptom
\end{tabular}




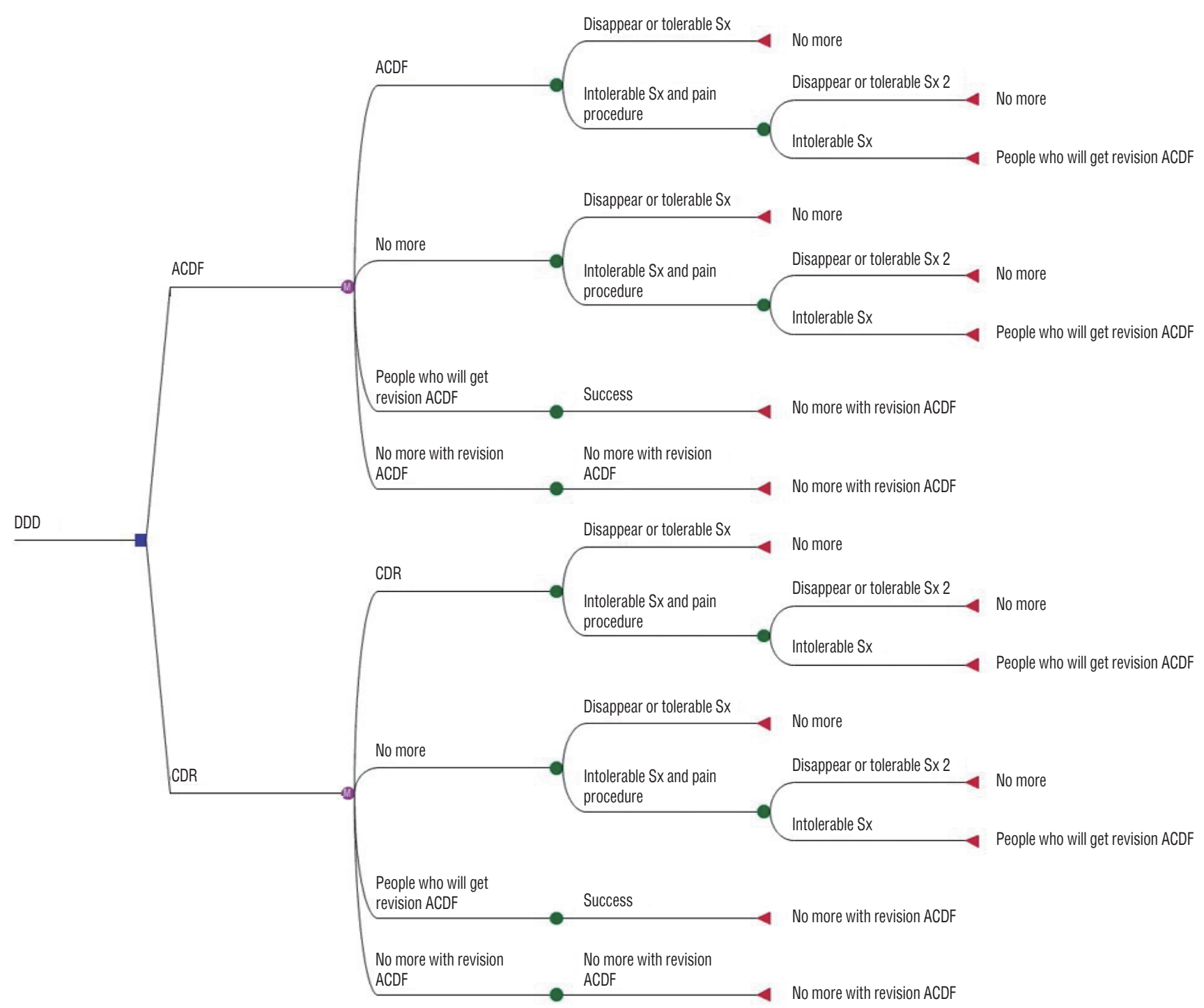

Fig. 1. Markarov model. DDD : degenerative disc disease, ACDF : anterior cervical discectomy and fusion, CDR : cervical disc replacement, Sx : symptom.

Table 3. Probability of transition to each health state

\begin{tabular}{llc}
\hline \multicolumn{1}{c}{ Health state } & $\begin{array}{c}\text { Probability of } \\
\text { transition (\%) }\end{array}$ \\
\hline ACDF & Disappear or tolerable Sx & 98.28 \\
& Intolerable Sx and pain procedure & 1.72 \\
& Disappear or tolerable Sx 2 & 87.5 \\
& People who had a revision ACDF & 12.5 \\
\hline CTDR & Disappear or tolerable Sx & 99.37 \\
& Intolerable Sx and pain procedure & 0.63 \\
& Disappear or tolerable Sx 2 & 50 \\
& People who had a revision ACDF & 50 \\
\hline
\end{tabular}

ACDF : anterior cervical discectomy and fusion, Sx : symptom, cTDR : cervical total disc replacement dition of the Markov model, was the reference of 0.7.

Qureshi et al. ${ }^{10)}$ reviewed the short form health survey (SF36) score, neck disability index (NDI) score, postoperative neurological improvement, ACDF and CDR results, and metatarsal analysis by comparing joint motility.

In this study, the utility weights of each health status were determined with reference to Qureshi et al. ${ }^{10)}$. The utility weights of each health condition in this study are shown in Table 5.

In this study, the assumptions set for the cost-benefit analysis through the Markov model are as follows. 1) ACDF and cTDR are two alternative methods for 40 -year-old patients who need surgical treatment for degenerative cervical disc 
Table 4. Medical costs

\begin{tabular}{lllc}
\hline & \multicolumn{1}{c}{ Surgical cost } & Procedure cost & Reoperation cost \\
\hline ACDF & KRW 4915420/USD 4632 & KRW 110583/USD 104 & KRW 6868233/USD 6472 \\
CTDR & KRW 7346799/USD 6923 & KRW 110583/USD 104 & KRW 6868233/USD 6472 \\
\hline
\end{tabular}

ACDF : anterior cervical discectomy and fusion, cTDR : cervical total disc replacement

Table 5. Cost-utility analysis results

\begin{tabular}{|c|c|c|c|c|c|}
\hline & Cost & Incremental cost & Utility (QALY) & Incremental utility (QALY) & Incremental cost-effectiveness ratio \\
\hline ACDF & $\begin{array}{c}\text { KRW } 2501807 \\
\text { USD } 2357\end{array}$ & & 3.72 & & \\
\hline CTDR & $\begin{array}{c}\text { KRW } 3685949 \\
\text { USD } 3473\end{array}$ & $\begin{array}{l}\text { KRW } 1184142 \\
\text { USD } 1116\end{array}$ & 4.18 & 0.46 & $\begin{array}{c}\text { KRW 2549511/QALY } \\
\text { USD 2402/QALY }\end{array}$ \\
\hline
\end{tabular}

QALY : quality adjusted life year, ACDF : anterior cervical discectomy and fusion, cTDR : cervical total disc replacement

disease. 2) The analysis period was 5 years, and the analysis cycle was 1 year. 3) Patients who underwent reoperation were assumed to have terminated treatment. 4) The number of times the procedure was performed was not considered. 5) The procedure was assumed to not affect quality of life. 6) Non-medical expenses and lost productivity costs were not considered. 7) The discount rate for future value was $3 \%$. And 8) health status weights used in previous US studies were applied.

No study subjects required further treatment after reoperation. Patients who underwent reoperation were assumed to have completed the treatment and remained in the revision ACDF health state.

\section{RESULTS}

The total cost of ACDF and cTDR for 40-year-old patients with ACDF or cTDR for 5 years was KRW 2501807/USD 2357 for ACDF and KRW 3685949/USD 3473 for cTDR. The cost difference between the two surgeries was KRW 1184142/USD 1116. Patients had 3.72 QALY after ACDF and 4.18 QALY after cTDR for 5 years. The difference between groups was 0.46 QALY. The cost-benefit analysis showed that the cumulative cost-effectiveness ratio of cTDR was KRW 2549511/QALY (USD 2,402/QALY) compared to ACDF. In this study, utility weights of 0.8 and 0.9 for ACDF and cTDR, respectively, were applied to the Markov model. The results of cost-utility analysis are shown in Table 5.

\section{DISCUSSION}

A cost-utility analysis is a special form of cost-effectiveness analysis. QALY or healthy years equivalents are indicators that reflect both quantitative aspects of life (life extension) and quality of life. Cost-utility analysis uses these two parameters as indicators of the outcome. The cost is measured in currency units. A cost-benefit analysis uses QALY, which reflects both quantitative and qualitative aspects of life and is the cost-effectiveness analysis, as compared to cost-effectiveness analysis, which uses only clinical results as quantitative aspects of life.

QALY is obtained by multiplying the life year by utility, which is the quality of life during survival. If the benefit to be generated in the future is smaller than the present benefit, the value of the future benefit should be reduced by a factor called the discount rate. The discounted value is called the present value. In the United States, many studies have been conducted at a discount rate of 3\%. Although QALY is difficult to measure consistently, QALY is recommended in various drug economic evaluation guidelines, including the Korean Health Insurance Review \& Assessment Service, since there is no appropriate alternative.

Both McAnany et al. ${ }^{9)}$ and Qureshi et al. ${ }^{10)}$ created Markov models and analyzed fictitious cohorts that were 45 and 40 years old, respectively, with analysis periods of 5 and 20 years respectively. The discount rate was 3\%. The utility weights were derived through different methods. In McAnany et al."), the utility weights of patients who received ACDF and cTDR were derived from questionnaires completed by 209 patients, 
and Qureshi et al. ${ }^{10)}$ derived utility weights from a meta-analysis of the literature.

As a result, in McAnany et al. ${ }^{9}$, the utility weight of patients receiving $\mathrm{ACDF}$ and $\mathrm{CTDR}$ was 0.72 , and the utility weight of patients receiving revision ACDF (reoperation ACDF) and revision cTDR (reoperation cTDR) was 0.43. In Qureshi et al. ${ }^{10}$, the utility weights were 0.8 for patients receiving ACDF, 0.9 for patients receiving CTDR, 0.75 for patients receiving revision ACDF, and 0.85 for patients receiving revision cTDR. It is unusual that the utility weights of patients who received ACDF and CTDR in McAnany et al. ${ }^{9)}$ were derived directly from the questionnaire, whereas the utility weights of patients receiving revision ACDF and revision CTDR were $60 \%$ of patients receiving ACDF and CTDR. Since the basis for this assumption is not mentioned in McAnany et al. ${ }^{9}$, we used the utility weights from Qureshi et al. ${ }^{10)}$.

Cost-benefit analyses of cTDR and ACDF have been conducted overseas, but those analyses differ from the Korean healthcare system. Therefore, those results cannot be applied to Korea. In this study, the cost and benefits were analyzed by applying the cost incurred to use the medical service in Korea and the incidence of postoperative complications (transition probability). Therefore, the results of this study may be more suitable for the Korean healthcare system than previous studies.

According to our data, the timing of the first ACDF or CTDR varied among patients. The timing of each reoperation and pain procedure differed and was difficult to apply to the model. Therefore, the probability of complications occurring each year was calculated and then reflected in the transition probability in the Markov model.

This study was conducted to evaluate the cost-effectiveness of ACDF and CTDR, which are mainly received by patients with degenerative cervical disc disease requiring surgical treatment. As a result, the incremental cost-effectiveness ratio of cTDR was KRW 2549511/QALY (USD 2402/QALY).

To choose a surgical procedure based on incremental costeffectiveness ratios, standards are needed. This criterion can be considered a societal willingness to pay. Generally, the costeffectiveness threshold is set as the cost limit that society will pay to extend 1 QALY. The World Health Organization aid that the cost per disability adjusted life year (DALY) is 'very cost-effective' when it is less than the per capita gross domestic product (GDP) of the country and is 'cost-effective' until the cost is less than three times the per capita GDP. Assuming that the standard of 1 DALY is the same as 1 QALY, the payment standard based on the Korean per capita GDP is about KRW 30899465/USD 29115.

Based on these payment criteria, the cumulative cost-effectiveness ratio of cTDR is KRW 2549511/QALY (USD 2402/QALY), which is less than KRW 30899465/USD 29115, so CTDR is more cost effective than ACDF. However, the costeffectiveness of cTDRs cannot be compared to ACDFs based solely on payment criteria.

When designing the research, we tried to use Korean National Health Insurance Corporation data or the Health Insurance Review \& Assessment Service data as representative data. However, because data satisfying all subject criteria were not available, data from one university general hospital were used. If the Korean National Health Insurance Corporation or Health Insurance Review \& Assessment Service data can be subdivided and the subjects classified in detail, the result will be more representative.

This study used utility weights from Qureshi et al. ${ }^{10)}$ study, which does not reflect differences between Americans and Koreans. There is a clear genetic and environmental difference between Americans and Koreans. Even after undergoing the same surgery, they may not have the same quality of life. Even though Americans and Koreans at similar levels can be evaluated differently, the utility weights of health state for Koreans should be collected and applied. To directly calculate the utility weights of health status, SF-36 and NDI data were collected and compared with ACDF and CTDR results, including mean hospital stay and a visual analog scale (VAS). However, the data might not be representative because the data collection period differed for each subject and many patients had no data. Therefore, the analysis was conducted with reference to US literature data. To obtain the utility weights of the health state for Koreans, systematic data collection will be conducted.

\section{CONCLUSION}

This study was a cost-benefit analysis of ACDF and cTDR, which are mainly performed for patients with degenerative cervical disc disease. Patients who received ACDF paid a total of KRW 2501807/USD 2357 over 5 years, yielding a utility of 3.72 QALY. Patients receiving cTDR paid a total of KRW 
3685949/USD 3473 over 5 years, yielding a benefit of 4.18 QALY. The cumulative effect ratio of cTDR compared to ACDF was KRW 2549511/QALY (USD 2402/QALY).

Both ACDF and cTDR are cost-effective alternatives for patients with degenerative cervical disc disease. On the other hand, CTDR is an effective option to get more benefits through additional costs.

\section{CONFLICTS OF INTEREST}

No potential conflict of interest relevant to this article was reported.

\section{INFORMED CONSENT}

Informed consent was obtained from all individual participants included in this study.

\section{References}

1. Anderson PA, Rouleau JP : Intervertebral disc arthroplasty. Spine (Phila Pa 1976) 29 : 2779-2786, 2004

2. Bohlman HH, Emery SE, Goodfellow DB, Jones PK : Robinson anterior cervical discectomy and arthrodesis for cervical radiculopathy. Longterm follow-up of one hundred and twenty-two patients. J Bone Joint
Surg Am 75 : 1298-1307, 1993

3. Cinotti G, David T, Postacchini $F$ : Results of disc prosthesis after a minimum follow-up period of 2 years. Spine (Phila Pa 1976) 21 : 995 1000, 1996

4. Coric D, Nunley PD, Guyer RD, Musante D, Carmody CN, Gordon CR, et al. : Prospective, randomized, multicenter study of cervical arthroplasty: 269 patients from the Kineflex|C artificial disc investigational device exemption study with a minimum 2-year follow-up: clinical article. J Neurosurg Spine $15: 348-358,2011$

5. Fryback DG, Dasbach EJ, Klein R, Klein BE, Dorn N, Peterson K, et al. : The Beaver Dam Health Outcomes Study: initial catalog of health-state quality factors. Med Decis Making $13: 89-102,1993$

6. Gold M : Panel on cost-effectiveness in health and medicine. Med Care 34 (12 Suppl) : DS197-DS199, 1996

7. Han SY, Kim HW, Lee CY, Kim HR, Park DH : Stand-alone cages for anterior cervical fusion: are there no problems? Korean J Spine 13 : 13-19, 2016

8. Kwon OI, Son DW, Lee SW, Song GS : Comparison of radiologic outcomes of different methods in single-level anterior cervical discectomy and fusion. Korean J Spine 13 : 91-96, 2016

9. McAnany SJ, Overley S, Baird EO, Cho SK, Hecht AC, Zigler JE, et al. : The 5-year cost-effectiveness of anterior cervical discectomy and fusion and cervical disc replacement: a Markov analysis. Spine (Phila Pa 1976) 39 : 1924-1933, 2014

10. Qureshi SA, McAnany S, Goz V, Koehler SM, Hecht AC : Cost-effectiveness analysis: comparing single-level cervical disc replacement and single-level anterior cervical discectomy and fusion: clinical article. J Neurosurg Spine $19:$ 546-554, 2013

11. Tengs TO, Wallace $A$ : One thousand health-related quality-of-life estimates. Med Care 38 : 583-637, 2000 